\title{
COVID-19 pandemic impact on clinical outcomes of patients with obstructive pyelonephritis
}

\author{
André B. Silva ${ }^{1}$ - Gustavo Freschi ${ }^{1} \cdot$ Raphael V. Carrera $^{1} \cdot$ Rafael H. Astolfi $^{1} \cdot$ Fernando F. Berti ${ }^{1} \cdot$ Nelson Gattas $^{1}$. \\ Alex Meller ${ }^{1}$ (1)
}

Received: 10 September 2020 / Accepted: 3 November 2020 / Published online: 21 November 2020

(c) Springer Nature B.V. 2020

\begin{abstract}
Purpose Obstructive pyelonephritis is a common urologic emergency that requires prompt decompression of the collecting system. The COVID-19 pandemic has changed patient flow and healthcare strategies at numerous emergency departments across Brazil with still unknown consequences for the population. This study sought to investigate the impact of the COVID19 outbreak on clinical outcomes in patients with acute obstructive pyelonephritis at a tertiary academic center.

Materials and methods After Institutional Review Board approval, a retrospective chart review of patients who required decompression of the collecting system due to acute obstructive pyelonephritis from June 2019 to July 2020 was conducted. Basic demographic information, pre-operative, and peri-operative data were recorded. Patients were assigned in "Pre-Covid" and "Post-Covid" groups based on the admission dates.

Results A total of 63 patients were included, with 40 patients in the Pre-Covid group and 23 in the Post-Covid group. Patients from the Post-Covid group presented at the ER later after symptoms onset ( 7.8 vs. 4.3 days; $p=0.012$ ), had higher rates of SIRS ( $57 \%$ vs. $25 \% ; p=0.012)$, perirenal abscesses ( $13 \%$ vs. $0 \% ; p=0.019)$, overall complications $(p=0.047)$ and presented longer hospital length of stay (7.6 vs. $3.8 ; p=0.007)$.

Conclusion During the COVID-19 pandemic, patients with acute obstructive pyelonephritis presented later for evaluation at the ER, had higher disease severity and longer hospital length of stay when compared to the pre-COVID group of patients with the same pathology.
\end{abstract}

Keywords Pyelonephritis · Coronavirus infection · Indwelling catheter $\cdot$ Systemic inflammatory response syndrome

$\begin{array}{ll}\text { Abbreviations } \\ \text { OPN } & \text { Obstructive pyelonephritis } \\ \text { ICU } & \text { Intensive care unit } \\ \text { ER } & \text { Emergency room } \\ \text { COVID-19 } & \text { Coronavirus disease } \\ \text { SIRS } & \text { Systemic inflammatory response syndrome } \\ \text { qSOFA } & \text { Quick sepsis-related organ failure } \\ & \text { assessment } \\ \text { DJ } & \text { Double-J stent }\end{array}$

Alex Meller

alexmeller@icloud.com

1 Department of Urology, Endourology Division, Federal University of São Paulo, Rua Napoleão de Barros, 715, 2nd floor, São Paulo, SP 04024-002, Brazil

\section{Introduction}

Obstructive pyelonephritis (OPN) is a very common clinical condition in urological practice [1], with high rates of sepsis and Intensive Care Unit (ICU) admissions [2, 3], leading to prolonged hospital length of stay and an overall mortality of $2 \%[4,5]$. Obstructive pyelonephritis is an emergency, where targeted treatment $[6,7]$ and prompt decompression of the collecting system $[8,9]$ can potentially improve clinical outcomes, with a high impact on patient morbidity and mortality $[10,11]$.

Concerns about the SARS-CoV-2 pandemic have been raised due to its high infectiousness, demanding radical changes in the basic structure of our present society [12]. Social distancing campaigns and a strict quarantine were implemented in numerous countries in an attempt to contain the COVID-19 dissemination [13, 14]. Brazil had the first confirmed case on February 26, 2020 and even before that, rumors about a potential pandemic were already impacting 
people's daily activities, as well as access to urgent healthcare. Even though it is difficult to assess the real impact of the COVID-19 on the healthcare system, several hospitals around the country have undergone radical changes to adapt to these new demands. Notably, there has been changes in patient flow at the emergency departments during the pandemic, and decreased hospital admissions due to cardiovascular diseases where reported by Huet et al. [15] in France, and Ebinger et al. [16] in the United States. In addition, ER visits for evaluation of patients with respiratory diseases increased dramatically during this period and consequently played a major role defining strategies as well as on how resources should be allocated to meet these new demands $[12,13]$.

In view of the intense changes evidenced in the routine of hospital care, we raised the hypothesis that patients with obstructive pyelonephritis are presenting themselves with greater severity due to the delay in seeking medical care. Thus, we sought to investigate the time elapsed between symptoms onset and medical care in this group of patients, as well as symptom severity when compared to patients with the same pathology outside the context of the pandemic.

\section{Materials and methods}

After Institutional Review Board approval, a retrospective chart review of patients who required decompression of the collecting system due to acute obstructive pyelonephritis from June 2019 to July 2020 was conducted. Basic demographic information, pre-operative, and peri-operative data were recorded. Patients were assigned in two groups: "Pre-Covid" and "Post-Covid". The latter was composed of patients admitted from February 26th, 2020 to July 2020. All patients admitted before February 26th, 2020 were included in the "Pre-Covid" group.

Acute obstructive pyelonephritis was defined as the presence of more than 5 white blood cells (WBC's)/high power field (hpf) in a centrifuged urinary specimen, an isolated bacterial count of $10^{4}$ colony-forming units $(\mathrm{CFU}) / \mathrm{mL}$ in the urine specimen, high-grade fever of more than $38^{\circ} \mathrm{C}$ in a patient with flank/lumbar pain and an obstructive ureteral stone confirmed by non-contrast CT scan. After diagnosis, patients were admitted in the ER for fluid resuscitation, broad-spectrum antibiotic therapy and emergent decompression of the collecting system using. According to the institutional protocol, an initial attempt of placing a double$\mathrm{J}$ stent was performed due to the lower patient morbidity and because it is readily available in the institution. After a failed attempt, a nephrostomy tube would be placed under ultrasound guidance.

In those cases where a renal abscess was present, a cutoff of size of $3 \mathrm{~cm}$ was used as a criterion when choosing between percutaneous drainage and conservative treatment. Refractory cases would be subject to further puncture or surgical drainage at the discretion of the assistant team. Patients with clinical or radiological findings of pulmonary involvement were systematically screened for COVID-19 utilizing a RT-PCR test from respiratory samples (e.g., nasopharynx), according to the Institutional Infection Control Commission.

Pre-operative data such as basic demographic information, comorbidities, time of initial symptoms onset, prior antibiotic usage, basic metabolic panel and C-reactive protein levels, radiological findings such as stone location, stone size, presence of hydronephrosis or perirenal abscess were collected. Information regarding postoperative complications, ICU admissions, time between admission and kidney decompression, hospital length of stay, as well as respiratory symptoms were assessed. SIRS was defined as the presence of at least two of the following criteria: body temperature $<36{ }^{\circ} \mathrm{C}$ or $>38{ }^{\circ} \mathrm{C}$, respiratory rate $>20$ breaths per minute or arterial $\mathrm{CO}_{2}$ tension $<32 \mathrm{mmHg}$, heart rate $>90$ beats per minute, white blood cell count $>12,000$ / $\mathrm{mm}^{3}$ or $<4000 / \mathrm{mm}^{3}$. The qSOFA score (respiratory rate $>22$ breaths per minute, Glasgow Coma Scale $<15$ and systolic arterial pressure $\leq 100 \mathrm{mmHg}$ ) was applied to all patients during the admission. Sepsis was defined as the presence of OPN and a qSOFA score $\geq 2$ [17]. Postoperative complications were graded according to the Clavien-Dindo classification [18].

The decision of admitting patients to the ICU was based on the diagnosis of OPN associated with hemodynamic instability or the presence of multiple comorbidities. After ICU discharge, patients presenting clinical stability and two consecutive drops in WBC's count and C-reactive protein levels were discharged with appropriate antibiotic regimen. Statistical analysis was performed using SPSS (V.9, SPSS Inc., Chicago, Illinois, USA). The Kolmogorov-Smirnov test and the histogram analysis were applied to assess the normal distribution of variables. Descriptive analyses were performed using Student's $t$ test or Mann-Whitney test for numerical variables and Chi-square to compare categorical variables. Significance was defined at $p \leq 0.05$.

\section{Results}

A total of 63 patients were included in our analysis, with 23 (36.5\%) patients in the Post-COVID group and 40 (63.5\%) in the Pre-COVID group. All patients were successfully managed with a double-J stent. Mean age and time since symptom onset were $46.8 \pm 13.4$ years and $5.5 \pm 4.1$ days, respectively. Mean length of stay was $5.2 \pm 4.8$ days. SIRS and sepsis were present in $23(36.5 \%)$ and $6(9.5 \%)$ patients, respectively, with an overall mortality rate of $1.5 \%$. 


\section{Post-COVID}

In the Post-COVID group, $56 \%$ of patients were females with a mean age of $47 \pm 15$ years and a mean time since symptoms onset of $7.8 \pm 5.5$ days. During the initial evaluation, $57 \%$ of patients had SIRS and $13 \%$ had sepsis. Mean time from initial evaluation until stent placement was $22.6 \pm 8.7 \mathrm{~h}$, with $48 \%$ of patients presenting pyuria immediately after kidney drainage. Basic demographic data, laboratorial and CT scan findings are described in Table 1. In the same group, three $(13 \%)$ patients were admitted at the intensive care unit postoperatively. Clavien $\geq 2$ complications were present in $26 \%$, with one patient requiring dialysis, one patient underwent nephrostomy tube placement due to persistent fever even after stent placement and one patient died after 24 days of admission. Mean length of stay in this group was $7.6 \pm 7$ days.

During the COVID-19 pandemic, two (8.6\%) patients presented at the ER with respiratory symptoms and one (4.3\%) had typical pulmonary findings on CT scan imaging. This patient required ICU admission, where he presented clinical worsening due to persistent obstruction of the collecting system even after the placement of the double-J stent, with the need of additional drainage utilizing a percutaneous nephrostomy tube. The patient showed progressive clinical improvement and was discharged after 25 days. No patient in this group was readmitted or had respiratory complications after 2 weeks of the initial evaluation.

\section{Pre-COVID}

The Pre-COVID group had 55\% of women, with a mean age of $46 \pm 12$ years. Mean time since symptoms onset was $4.3 \pm 2.4$ days and $52 \%$ of patients presented fever at the initial evaluation. SIRS and sepsis were present in $25 \%$ and $7 \%$ of patients, respectively. Mean time since the initial evaluation and kidney decompression was $23.7 \pm 10 \mathrm{~h}$, with the presence of pyuria in $37 \%$ of the cases after double-J placement. Basic demographic and complementary data are shown in Table 1 . In this group, 4 (10\%) of patients required ICU admission postoperatively due to hemodynamic instability after kidney decompression. Despite that, all patients showed progressive clinical improvement and were discharged within a week of hospitalization. Furthermore, four (10\%) patients presented Clavien $\geq 2$ complications, with the need of broad-spectrum antibiotics in three patients and hemodialysis in one case. Mean length of stay for this group was $3.8 \pm 2$ days and no patient required a new hospitalization within 2 weeks of discharge.

\section{Outcomes}

Among the 23 patients who underwent surgery during the pandemic, only $1(4.3 \%)$ had a confirmed diagnose of COVID-19 concomitantly with obstructive pyelonephritis, and no correlation between the COVID-19 and symptom severity $(p=0.334)$ was established. Patients from the PostCOVID group sought medical care with a longer history of symptoms (7.8 vs. 4.3 days; $p=0.012$ ), had a higher incidence of SIRS on admission (57 vs. $25 \% ; p=0.012$ ) and a higher incidence of perirenal abscesses on CT scan (13 vs. $0 \% ; p=0.019)$. Only one of the three cases presenting renal abscesses required percutaneous drainage. All patients in this group had a good clinical outcome, with a mean hospital stay of 4.6 days. The Post-COVID group also had higher overall complication rates ( $p=0.047$; Fig. 1$)$ and longer length of stay ( 7.6 vs. 3.8 days; $p=0.007$ ). Comparative data are summarized in Table 2.

\section{Discussion}

The COVID-19 pandemic significantly impacted the healthcare system in Brazil, not only because of the escalating demand from patients infected by the virus, but also due to a sudden decrease in the availability of resources for diagnosis and treatment of numerous other diseases. Emergency departments across the country were also affected and deserve a closer look, as a significant portion of patients presenting at the ER demand a faster course of action from the medical team to improve clinical outcomes and reduce inpatient mortality [19]. Obstructive pyelonephritis is a common and potentially fatal condition that requires prompt intervention with antibiotics and decompression of the collecting system. To date, the real impact of the COVID-19 on the urinary tract is still not clear, but recent reports showed kidney dysfunction in $0.1-29 \%$ of cases and increased mortality rates within this group of patients $(60-90 \%)$ [20].

Previously to the pandemic, studies reported a mean time between symptom onset and hospital admission of 2-3 days, with a positive correlation between this variable and the incidence of septic shock [21, 22]. In this study, patients from the Post-COVID group had a longer interval of time since symptom onset and hospital admission (7.8 vs. 4.3 days; $p=0.012$ ). We estimate that this delay is probably a consequence of the strict social distancing measures imposed during the pandemic and the fear from a potential infection by the virus during the medical evaluation at the ER.

In a recent study by Fukushima et al. [23] prior to the COVID-19 pandemic, the authors reported a SIRS rate of $72 \%$ in patients with obstructive pyelonephritis. Our results showed a greater severity in patients who presented OPN during the pandemic, with higher incidence of SIRS (57 
Table 1 Demographic and clinical data

\begin{tabular}{|c|c|c|c|}
\hline & COVID era $(n=23)$ & Non-COVID era $(n=40)$ & $p$ \\
\hline \multicolumn{4}{|l|}{ Sex } \\
\hline Male & $10(44 \%)$ & $18(45 \%)$ & \multirow[t]{2}{*}{0.907} \\
\hline Female & $13(56 \%)$ & $22(55 \%)$ & \\
\hline Age (years) & $47 \pm 15$ & $46 \pm 12$ & 0.862 \\
\hline \multicolumn{4}{|l|}{ Charlson score } \\
\hline 0 & $16(70 \%)$ & $30(75 \%)$ & \multirow[t]{3}{*}{0.687} \\
\hline 1 & $4(17 \%)$ & $4(10 \%)$ & \\
\hline 2 & $2(9 \%)$ & $6(15 \%)$ & \\
\hline 3 & $1(4 \%)$ & & \\
\hline Time since symptoms onset (days) & $7.8 \pm 5.5$ & $4.3 \pm 2.4$ & 0.012 \\
\hline \multicolumn{4}{|l|}{ Fever } \\
\hline Yes & $11(47.8 \%)$ & $21(52 \%)$ & \multirow[t]{2}{*}{0.721} \\
\hline No & $12(52.2 \%)$ & $19(48 \%)$ & \\
\hline WBC's $\left(10^{3} / \mu \mathrm{L}\right)$ & $17.3 \pm 7.7$ & $16.1 \pm 5.1$ & 0.567 \\
\hline \multicolumn{4}{|l|}{ WBC's left shift } \\
\hline Yes & $13(56.5 \%)$ & $22(55 \%)$ & \multirow[t]{2}{*}{0.907} \\
\hline No & $10(43.5 \%)$ & $18(45 \%)$ & \\
\hline $\mathrm{CRP}(\mathrm{mg} / \mathrm{dL})$ & $142 \pm 119$ & $200 \pm 151$ & 0.125 \\
\hline Serum creatinine $(\mathrm{mg} / \mathrm{dL})$ & $1.58(1.2-2.43)$ & $1.5(1.02-2.3)$ & 0.822 \\
\hline \multicolumn{4}{|l|}{ Previous antibiotic usage } \\
\hline Yes & $6(26 \%)$ & $6(15 \%)$ & \multirow[t]{2}{*}{0.281} \\
\hline No & $17(74 \%)$ & $34(85 \%)$ & \\
\hline \multicolumn{4}{|l|}{ Previous uroculture } \\
\hline Positive & $6(26 \%)$ & $12(30 \%)$ & \multirow[t]{2}{*}{0.741} \\
\hline Negative & $17(74 \%)$ & $28(70 \%)$ & \\
\hline \multicolumn{4}{|l|}{ Nitrite } \\
\hline Positive & $9(39 \%)$ & $9(23 \%)$ & \multirow[t]{2}{*}{0.159} \\
\hline Negative & $14(61 \%)$ & $31(77 \%)$ & \\
\hline \multicolumn{4}{|l|}{ Urine WBC's } \\
\hline Present & $20(87 \%)$ & $32(80 \%)$ & \multirow[t]{2}{*}{0.484} \\
\hline Absent & $3(13 \%)$ & $8(20 \%)$ & \\
\hline \multicolumn{4}{|l|}{ SIRS } \\
\hline Present & $13(57 \%)$ & $10(25 \%)$ & \multirow[t]{2}{*}{0.012} \\
\hline Absent & $10(43 \%)$ & $30(75 \%)$ & \\
\hline \multicolumn{4}{|l|}{ qSOFA } \\
\hline 0 & $9(39 \%)$ & $27(68 \%)$ & \multirow[t]{4}{*}{0.137} \\
\hline 1 & $11(48 \%)$ & $10(25 \%)$ & \\
\hline 2 & $1(4 \%)$ & $2(5 \%)$ & \\
\hline 3 & $2(9 \%)$ & $1(2 \%)$ & \\
\hline \multicolumn{4}{|l|}{ Hydronephrosis } \\
\hline Absent & $2(9 \%)$ & $14(35 \%)$ & 0.289 \\
\hline Mild & $8(35 \%)$ & $23(58 \%)$ & \\
\hline Moderate & $11(47 \%)$ & $3(7 \%)$ & \\
\hline Severe & $2(9 \%)$ & & \\
\hline Perirenal fat stranding & & & \\
\hline Present & $16(70 \%)$ & $28(70 \%)$ & 0.971 \\
\hline Absent & $7(30 \%)$ & $12(30 \%)$ & \\
\hline Abscess & & & \\
\hline Present & $3(13 \%)$ & $0(0 \%)$ & 0.019 \\
\hline Absent & $20(87 \%)$ & $40(100 \%)$ & \\
\hline Stone size $(\mathrm{mm})$ & $8(7-10)$ & $8(6-11)$ & 0.592 \\
\hline
\end{tabular}

Significant results are indicated in bold

$W B C$ white blood cells, $C R P$ C-reactive protein 


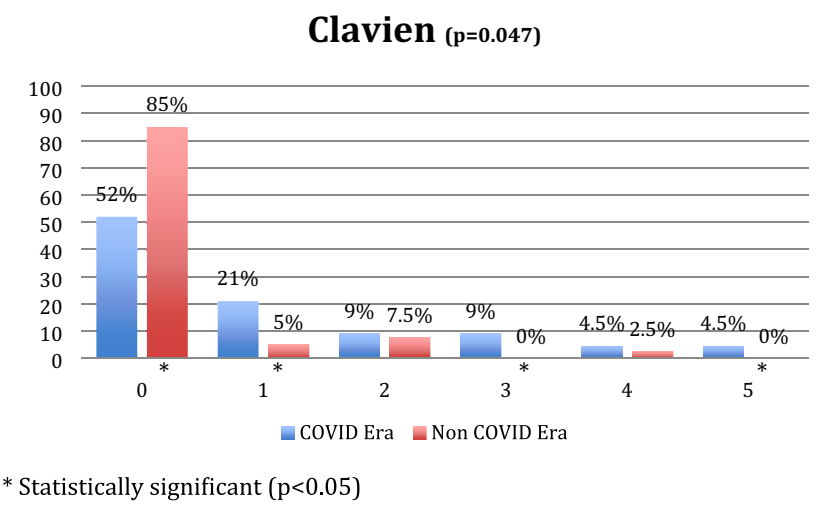

Fig. 1 Clavien-Dindo classification

Table 2 Results

\begin{tabular}{|c|c|c|c|}
\hline & COVID era $(n=23)$ & $\begin{array}{l}\text { Non-COVID } \\
\text { era }(n=40)\end{array}$ & $p$ \\
\hline DJ time $^{\mathrm{a}}(\mathrm{h})$ & $22.6 \pm 8.7$ & $23.7 \pm 10$ & 0.594 \\
\hline \multicolumn{4}{|l|}{ ICU } \\
\hline Yes & $3(13 \%)$ & $4(10 \%)$ & \multirow[t]{2}{*}{0.71} \\
\hline No & $20(87 \%)$ & $36(90 \%)$ & \\
\hline \multicolumn{4}{|l|}{ Pyuria after drainage } \\
\hline Yes & $11(48 \%)$ & $15(37 \%)$ & \multirow[t]{2}{*}{0.423} \\
\hline No & $2(52 \%)$ & $25(63 \%)$ & \\
\hline \multicolumn{4}{|l|}{ Clavien-Dindo } \\
\hline$<2$ & $17(74 \%)$ & $36(90 \%)$ & \multirow[t]{2}{*}{0.09} \\
\hline$\geq 2$ & $6(26 \%)$ & $4(10 \%)$ & \\
\hline Hospital stay (days) & $7.6 \pm 7$ & $3.8 \pm 2$ & 0.007 \\
\hline
\end{tabular}

Significant results are indicated in bold

${ }^{a} D J$ time: time from admission until surgery

vs. $25 \% ; p=0.012)$ and perirenal abscesses (13 vs. $0 \%$; $p=0.019)$ at admission when compared to the control group. Renal or perirenal abscesses are uncommon conditions with an incidence from 1 to 10 per 10,000 hospitalizations in the United States [24], but are usually associated chronic diseases [25] and therefore it could explain the higher incidence of these findings in the Post-COVID group. White blood cells count and CRP were previously associated with higher patient severity, as described by Flukes and Yamamoto [21, 23, 26]. However, our analysis did not find any correlation between these two parameters and worse clinical outcomes (WBC 17.3 vs. $16.1 \times 10^{3} / \mu \mathrm{L} ; p=0.567$ and CRP 142 vs. $200 \mathrm{mg} / \mathrm{dL} ; p=0.125$ ).

Earlier studies reported ICU admission rates of 7-17\% for patients with APN [24-27]. This study presented an overall ICU admission rate of $13 \%$, with no differences between groups. However, the lack of a sufficient number of ICU beds in the Brazilian public health system is a possible confounding factor, aggravated by the redistribution of a large percentage of ICU beds to patients with complications of the COVID-19 infection. This could justify the similar rates regarding ICU admission between the two groups, despite the greater severity of patients from the Post-COVID group. These patients presented higher rates of clinically significant complications (Clavien $\geq 2$ ), with a total of $6(26 \%)$ cases. Historically, hospital lengths of stay of patients with OPN vary from 4.25 to 12 days $[22,27,1]$. This study demonstrated that patients from the Post-Covid group had a significantly higher length of stay when compared to the Pre-Covid group ( 7.6 vs. 3.8 days; $p=0.007$ ), possibly due to the higher Clavien $\geq 2$ complication rates in the first group.

A positive correlation between COVID-19 and OPN still lacks evidence, and the limited availability of tests to detect infected patients in this study precludes any causality analysis. Nonetheless, public health measures created to mitigate the impact of the pandemic ultimately resulted in delayed hospital admissions and possibly contributed to the higher severity of the Post-Covid group of patients. The novel coronavirus is a serious public health issue, which requires a coordinated course of action to optimize resource allocation without compromising patient care. Efforts should be made to improve patient inflow at the ER to identify patients with suspected OPN and avoid any treatment delay, which could impact patient outcomes and increase mortality.

This study has some limitations. First, the limited number of patients and a retrospective design preclude more accurate conclusions and limit the generalizability of the results. However, we presented the first report on patients with obstructive pyelonephritis at a high volume tertiary academic center located at the largest city in Brazil. In an era of ever-growing medical knowledge concerning COVID19 , we provided preliminary information that can contribute to the development of protocols for patients with suspected OPN. Second, only three patients in the study were tested for COVID-19, mainly due to established governmental protocols that did not recommend mandatory screening for COVID in asymptomatic patients presenting at the ER in an attempt to avoid test shortage. Thus, it is impossible to determine whether there were asymptomatic carriers of the virus and if this had influenced the severity of cases of OPN in the Post-COVID group.

COVID-19 is a serious public health problem, which deserves all the care inherent to tragedies of this magnitude. However, it should not delay or postpone the treatment of potentially serious conditions such as Obstructive Pyelonephritis. Studies like ours serve as a warning to health professionals and public managers, who must be alert and implement measures that minimize the side effects of the pandemic. 
In conclusion, during the COVID-19 pandemic, patients with acute obstructive pyelonephritis presented themselves at the emergency room with a longer interval of time since symptoms onset and with greater disease severity. In addition, length of hospitalization was longer for this group of patients when compared to patients with OPN prior to the pandemic.

Author contributions Silva (first author): data collection, data analysis, and manuscript writing. Freschi (Co-corresponding author): protocol development and manuscript editing. Meller (Co-corresponding author): manuscript editing. Astolfi: manuscript editing. Berti: manuscript editing. Carrera: manuscript editing. Gattas: manuscript editing.

Funding None.

\section{Compliance with ethical standards}

Conflict of interest The authors declare that they have no conflict of interest.

\section{References}

1. Pandey S, Sankhwar SN, Goel A et al (2019) Quick sequential (sepsis related) organ failure assessment: a high performance rapid prognostication tool in patients having acute pyelonephritis with upper urinary tract calculi. Investig Clin Urol 60(2):120-126. https://doi.org/10.4111/icu.2019.60.2.120

2. Hsu CY, Fang HC, Chou KJ, Chen CL, Lee PT, Chung HM (2006) The clinical impact of bacteremia in complicated acute pyelonephritis. Am J Med Sci 332:175-180. https://doi. org/10.1097/00000441-200610000-00004

3. Naber KG (2006) Urogenital infections: the pivotal role of the urologist. Eur Urol 50:657. https://doi.org/10.1016/j.eurur o.2006.03.056

4. Yoshimura K, Utsunomiya N, Ichioka K et al (2005) Emergency drainage for urosepsis associated with upper urinary tract calculi. J Urol 173:458. https://doi.org/10.1097/01.ju.0000150512 $.40102 . b b$

5. Hamasuna R, Takahashi S, Nagae H et al (2015) Obstructive pyelonephritis as a result of urolithiasis in Japan: diagnosis, treatment and prognosis. Int J Urol 22:294. https://doi. org/10.1111/iju.12666

6. Bone RC, Balk RA, Cerra FB et al (1992) Definitions for sepsis and organ failure and guidelines for the use of innovative therapies in sepsis. The ACCP/SCCM Consensus Conference Committee. American College of Chest Physicians/Society of Critical Care Medicine. Chest 101:1644-1655. https://doi. org/10.1378/chest.101.6.1644

7. Singer M, Deutschman CS, Seymour CW et al (2016) The third international consensus definitions for sepsis and septic shock (sepsis-3). JAMA 315:801. https://doi.org/10.1001/ jama.2016.0287

8. Türk C, Neisius A, Petrik A et al (2020) Urolithiasis guidelines panel. EAU-ESTRO-ESUR-SIOG guidelines urolithiasis. Eur Urol. 978-94-92671-07-3.

9. Assimos D, Krambeck A, Miller NL et al (2016) Surgical management of stones: American Urological Association/Endourological Society Guideline. J Urol 196(4):1153-1160. https://doi. org/10.1016/j.juro.2016.05.090
10. Borofsky MS, Walter D, Shah O et al (2013) Surgical decompression is associated with decreased mortality in patients with sepsis and ureteral calculi. J Urol 189:946. https://doi. org/10.1016/j.juro.2012.09.088

11. Pearle MS, Pierce HL, Miller GL et al (1998) Optimal method of urgent decompression of the collecting system for obstruction and infection due to ureteral calculi. J Urol 160:1260. https:// doi.org/10.1016/S0022-5347(01)62511-4

12. Zylke JW, Bauchner H (2020) Mortality and morbidity: the measure of a pandemic. JAMA. Published online July 01, 2020. https://doi.org/10.1001/jama.2020.11761

13. World Health Organization. Coronavirus disease (COVID-19) outbreak.

14. Doglietto F, Vezzoli M, Gheza F, et al (2019) Factors associated with surgical mortality and complications among patients with and without coronavirus disease 2019 (COVID-19) in Italy. JAMA Surg. Published online June 12, 2020. https://doi. org/10.1001/jamasurg.2020.2713

15. Huet F, Prieur C, Schurtz G et al (2020) One train may hide another: Acute cardiovascular diseases could be neglected because of the COVID-19 pandemic. Arch Cardiovasc Dis 113(5):303-307. https://doi.org/10.1016/j.acvd.2020.04.002

16. Ebinger JE, Shah PK (2020) Declining admissions for acute cardiovascular illness: the COVID-19 paradox. J Am Coll Cardiol 76(3):289-291. https://doi.org/10.1016/j.jacc.2020.05.039

17. Seymour CW, Liu VX, Iwashyna TJ et al (2016) Assessment of clinical criteria for sepsis for the third international consensus definitions for sepsis and septic shock (sepsis-3). JAMA 315(8):762-774. https://doi.org/10.1001/jama.2016.0288

18. Dindo D, Demartines N, Clavien PA (2004) Classification of surgical complications: a new proposal with evaluation in a cohort of 6336 patients and results of a survey. Ann Surg 240(2):205213. https://doi.org/10.1097/01.sla.0000133083.54934.ae

19. Puliatti S, Eissa A, Eissa R et al (2020) COVID-19 and urology: a comprehensive review of the literature. BJU Int 125(6):E7E14. https://doi.org/10.1111/bju.15071

20. Naicker S, Yang CW, Hwang SJ, Liu BC, Chen JH, Jha V (2020) The novel coronavirus 2019 epidemic and kidneys. Kidney Int 97(5):824-828. https://doi.org/10.1016/j.kint.2020.03.001

21. Yamamoto Y, Fujita K, Nakazawa S et al (2012) Clinical characteristics and risk factors for septic shock in patients receiving emergency drainage for acute pyelonephritis with upper urinary tract calculi. BMC Urol 12:4. https://doi. org/10.1186/1471-2490-12-4

22. Tambo M, Okegawa T, Shishido T, Higashihara E, Nutahara K (2014) Predictors of septic shock in obstructive acute pyelonephritis. World J Urol 32(3):803-811. https://doi.org/10.1007/ s00345-013-1166-4

23. Fukushima H, Kobayashi M, Kawano K, Morimoto S (2018) Performance of quick sequential (sepsis related) and sequential (sepsis related) organ failure assessment to predict mortality in patients with acute pyelonephritis associated with upper urinary tract calculi. J Urol 199(6):1526-1533. https://doi. org/10.1016/j.juro.2017.12.052

24. Rubilotta E, Balzarro M, Lacola V, Sarti A, Porcaro AB, Artibani W (2014) Current clinical management of renal and perinephric abscesses: a literature review. Urologia 81(3):144-147. https://doi.org/10.5301/urologia.5000044

25. Coelho RF, Schneider-Monteiro ED, Mesquita JL, Mazzucchi E, Marmo Lucon A, Srougi M (2007) Renal and perinephric abscesses: analysis of 65 consecutive cases. World J Surg 31(2):431-436. https://doi.org/10.1007/s00268-006-0162-x

26. Flukes S, Hayne D, Kuan M, Wallace M, McMillan K, Rukin NJ (2015) Retrograde ureteric stent insertion in the management of infected obstructed kidneys. BJU Int 115(5):31-34. https://doi. org/10.1111/bju.12918 
27. Pricop C, Puia D, Mereuta O et al (2016) Infected hydronephrosis: can we reduce patient suffering and costs? J Pak Med Assoc 66(11):1372-1377
Publisher's Note Springer Nature remains neutral with regard to jurisdictional claims in published maps and institutional affiliations. 